\title{
Room for the candidate gene approach in the genome-wide association era: an example from GSTP1 gene polymorphism
}

\author{
Wojciech Mlynarski
}

Department of Paediatrics, Oncology, Haematology and Diabetology, Medical University of Lodz, Poland

Submitted: 28 August 2012

Accepted: 28 August 2012

Arch Med Sci 2012; 8, 4: 606-607

DOI: 10.5114/aoms.2012.30301

Copyright @ 2012 Termedia \& Banach

The evidence for a role of genetic factors in susceptibility to common, multifactorial type 2 diabetes (T2DM) has been known for decades. It has come from twin studies and the analysis of the relative risk in families with affected individuals. After the first successes in monogenic diabetes were published in the early 1990s, the scientists started their effort to identify genes for complex T2DM. They soon realized that this task was much more difficult to achieve than the routine procedure of cloning genes for Mendelian diseases. This was a consequence of the fact that the phenotype of T2DM was, in addition to environmental factors, the result of the complex interaction between tens or even hundreds of variants with very low relative risk located in different genes. In the early years of the research on the genetics of T2DM two approaches were used. First, the researchers looked for an association between frequent polymorphism in the candidate genes and the disease. The candidate genes were selected based on their role in insulin secretion or insulin action. The basic notion of association studies was that susceptibility alleles should be more prevalent in the cases than in the controls. Those early studies suffered from a lack of statistical power, the positive results were difficult to replicate, and soon it became apparent that our knowledge on the best candidates was not excellent. The second approach, linkage analysis, was associated with the idea of positional cloning that worked well for monogenic diseases. In complex traits, however, with the small sizes of the families, a very modest effect of each gene and difficulties to establish the critical interval, this approach brought a lot of disappointments. In fact the list of T2DM genes successfully identified over the decade beginning in the mid 1990s contained just three positions: PPAR- $\gamma$ (PPARG) and Kir6.2 (KCN/11) found by the candidate gene approach and TCF7L2 discovered by the positional cloning technique [1]. The recent years have given us a new tool to discover genetic susceptibility to T2DM, e.g. genome-wide association studies (GWAS). This progress was associated with new technologies that were able to generate a lot of genotyping and the advances in our understanding of the organisation and physiology of human DNA. The scientists have also learned their lessons and they understood that large groups of patients and controls are necessary. The GWAS finally produced major advances in the field of genetics of complex T2DM and many other common diseases.

\author{
Corresposponding author: \\ Prof. Wojciech Mlynarski MD, PhD \\ Department of Paediatrics, \\ Oncology, Haematology \\ and Diabetology \\ Medical University of Lodz \\ 36/50 Sporna St \\ 91-738 Lodz, Poland \\ Phone: +4842617 7769 \\ E-mail: \\ Wojciech.Mlynarski@umed.lodz.pl
}


This approach has facilitated a substantial and rapid rise in the number of confirmed genetic susceptibility variants for T2DM.

The first GWAS for T2DM was conducted in a French cohort. This study identified novel association signals at SLC3OA8, HHEX, LOC387761 and EXT2, and validated the previously identified association at TCF7L2 [2]. Nowadays, most of the T2DM genetic cohorts have combined to form DIAGRAM+, which yields an effective sample size of more than 22,000 subjects of European origin. In a recent study, 2,426,886 imputed and genotyped autosomal single nucleotide polymorphisms (SNPs), with additional interrogation of the $\mathrm{X}$ chromosome, were examined for association with T2DM. More than 50 variants have been identified so far, many of which were discovered through GWAS [3].

However, there are several limitations of GWAS. First, there are a considerable number of uncaptured SNPs in the public database, and there has been insufficient effort to perform GWAS for populations other than those of European origin. The rationale of GWAS is based on the "common disease-common variant" hypothesis, and studies have focused on finding common variants associated with the disease; therefore, susceptibility variants having a minor allele frequency (MAF) of less than $1 \%$ are frequently missed. Moreover, results collected from GWAS cannot be generalized since some genetic variants and predispositions may be population-specific. In the light of these limitations there is still room for the candidate gene approach in the GWAS era [4].

Growing evidence indicates that oxidative stress is increased in diabetes due to overproduction of reactive oxygen species and decreased efficiency of antioxidant defences. Recently some papers have indicated that oxidative stress may play a role in pathogenesis of pancreatic $\beta$ cell destruction and type 2 diabetes. Among known players glutathione S-transferases (GSTs) are phase II detoxifying enzymes that catalyze the conjugation of reduced glutathione to a wide range of electrophilic substrates and represent a major protective mechanism against oxidative stress. Epidemiological studies indicate that individuals with the GSTP1 protein with valine rather than isoleucine at position 105 (GSTP1 Ile105Val) have significantly decreased catalytic efficiency of this enzyme [5]. A study published in the current issue of Archives of Medical Science reports an association between GSTP1 genotype and T2DM in the Egyptian population [6]. The study was conducted on a relatively small group of 112 T2DM patients and 188 matched controls. They found that the $\mathrm{Val}$ allele is a risk factor for developing T2DM. To strengthen this observation they also reported a weak association with increase in body mass index (BMI) within the study group.
In the literature there are ambiguous results showing no association between GSTP1 genotype and T2DM or even an opposite effect of Ile105Val variants $[7,8]$. Thus, large, well-designed studies with appropriate statistical power are needed to confirm these results in this special population of Egyptian patients with type 2 diabetes. However, this study has pointed out a possible link between type 2 diabetes and oxidative stress as a new pathway to future targeted therapy. Furthermore, the weak genetic association found in the study is a nice example of the need for the candidate gene approach in the GWAS era.

\section{Acknowledgments}

The author is funded by the Innovative Economy Operational Programme - Activity 1.2 (TEAM Programme coordinated by the Foundation for Polish Science).

\section{References}

1. Malecki MT, Mlynarski W, Skupien J. Can geneticists help clinicians to understand and treat non-autoimmune diabetes? Diabetes Res Clin Pract 2008; 82 Suppl 2: S83-93.

2. Sladek R, Rocheleau G, Rung J, et al. A genomewide association study identifies novel risk loci for type 2 diabetes. Nature 2007; 445: 881-5.

3. Voight BF, Scott LJ, Steinthorsdottir V, et al. MAGIC investigators; GIANT Consortium. Twelve type 2 diabetes susceptibility loci identified through large-scale association analysis. Nat Genet 2010; 42: 579-89.

4. Imamura M, Maeda S. Genetics of type 2 diabetes: the GWAS era and future perspectives. Endocr J 2011; 58:723-39

5. Kariž S, Nikolajević Starčević J, Petrovič D. Association of manganese superoxide dismutase and glutathione S-transferases genotypes with myocardial infarction in patients with type 2 diabetes mellitus. Diabetes Res Clin Pract 2012 [Epub ahead of print].

6. Amer MA, Ghattas MH, Abo-ElMatty DM, Abou-El-Ela SH. Evaluation of glutathione S-transferase P1 genetic variants affecting type-2 diabetes susceptibility and glycemic control. Arch Med Sci 2012; 8: 631-6.

7. Gönül N, Kadioglu E, Kocaba NA, Ozkaya M, Karakaya AE, Karahalil B. The role of GSTM1, GSTT1, GSTP1, and OGG1 polymorphisms in type 2 diabetes mellitus risk: a casecontrol study in a Turkish population. Gene 2012; 505: 121-7.

8. Bid HK, Konwar R, Saxena M, Chaudhari P, Agrawal CG, Banerjee M. Association of glutathione S-transferase (GSTM1, T1 and P1) gene polymorphisms with type 2 diabetes mellitus in north Indian population. J Postgrad Med 2010; 56: 176-81. 